\title{
Antisense oligonucleotide p45Skp-2 suppresses migratory chemotactic and metastasis of oral malignant Burkitt's lymphoma cell through down-regulation of MTA-1 and induction of E-cadherin mechanism
}

\author{
Supriatno ${ }^{1 *}$, Dyah Irnawati ${ }^{1}$, Ana Medawati ${ }^{3 *}$ \\ ${ }^{1}$ Department of Oral Medicine, Faculty of Dentistry Gadjah Mada University, Indonesia \\ ${ }^{2}$ Department of Dental Biomaterial, Faculty of Dentistry Gadjah Mada University, Indonesia \\ ${ }^{3}$ Post Graduate Program, Faculty of Dentistry Gadjah Mada University, Indonesia
}

\begin{abstract}
Introduction: Burkitt's lymphoma is a high-grade B-cell neoplasm and one of the most aggressive malignancies of lymphoid origins which found mainly in the paediatric population. The treatment options of this tumour are still limited. However, a new strategy for refractory tumour, phosphorothioate oligonucleotide antisense technique has watched with keen interest. This study was aimed to examine the effect of antisense p45Skp-2 (Skp-2 AS) suppressed migratory chemotactic and metastasis of oral malignant Burkitt's lymphoma (Raji) cell through down-regulation of MTA-1 and E-cadherin. Methods: True experiment laboratory with post-test control group design was confirmed in this study. The efficiency of Skp-2 AS in the suppression of cell chemotactic migration was examined by Boyden chamber assay. To evaluate the inhibition of cell metastasis was conducted by decreasing MTA-1 expression protein. The expressions of MTA-1, E-cadherin and a-tubulin protein were investigated by Western blot analysis. Results: The results revealed that the number of chemotactic migration of Skp-2AS treated Raji cell was significantly decreased when compared with that of sense p45Skp-2 (Skp-2S) and scrambled control (SC) cells $(P<0.05)$ followed by decreased expressions of MTA-1 protein and overexpression of E-cadherin. Interestingly, the expression of a-tubulin protein as an internal control was approximately similar in each transfectant cells. Conclusion: p45Skp-2 have an antitumor activity via suppression of migratory chemotactic activity and metastasis on oral Burkitt's lymphoma cells through down-regulation of MTA-1 and induction of E-cadherin proteins targeting this molecule could represent a promising new therapeutic approach for this type of cancer.
\end{abstract}

Keywords: Burkitt's lymphoma cell, Skp-2 AS, MTA-1, E-cadherin, chemotactic migration, metastatic.

p-ISSN: 1979-0201; e-ISSN: 2549-6212; Available from: http://jurnal.unpad.ac.id/pid/article/view/21756

DOI: 10.24198/pid.vol32no3.21756

Submission: Nov 28, 2019; Accepted: Oct 1, 2020; Published online: Nov 30, 2020

"Corresponding author: Supriatno, Department of Oral Medicine, Faculty of Dentistry Gadjah Mada University, Indonesia. 1, Jalan Denta, Yogyakarta, Special Region of Yogyakarta, Indonesia, 55281. Phone: +6281931363797; Email: pridentagama@ gmail.com 


\section{INTRODUCTION}

S-phase kinase-associated protein-2 (p45Skp-2 or Skp-2) is a ubiquitin ligase complex of Skp, Cullin, F-box (SCF)-Skp-2. ${ }^{1}$ Skp-2 plays a role in the ubiquitin-mediated degradation of the cyclin-dependent kinase (CDK) inhibitor p27Kip1 and positively regulates the $\mathrm{G}_{1}$-to-S transition. ${ }^{2,3}$ Decreased Skp2 expression leads to accumulation of p27 and induces cell cycle arrest in the $G_{1}$ phase. Skp2 is required by other cell-cycle regulators, such as free cyclin-E, ${ }^{4} \mathrm{E} 2 \mathrm{~F} 1,{ }^{5}$ and hOrclp, for ubiquitination ${ }^{6}$. Up-regulation of Skp2 has been observed in various types of human cancer. Overexpression of Skp-2 indicates a poor prognosis and aggressive disease among patients with colorectal, ${ }^{7}$ hypupharingeal ${ }^{8}$, gastric, ${ }^{9}$ and pancreatic cancers ${ }^{2}$ Skp2 knock-out (-/-) mice show smaller organs and slower growth, and their cells exhibit induced apoptosis. ${ }^{4}$ Despite these findings, however, little is known about the mechanism of Skp-2 overexpression in cancer cells or the nature of the contribution of this protein to the malignant phenotype.

Burkitt's lymphoma (BL) is a high-grade and aggressive malignancy of B-cell lymphoid origin that occurs as $3 \%-5 \%$ of all lymphomas. BL occurs in many groups of children, $40 \%$ of whom suffer from non-Hodgkin's lymphoma. ${ }^{10}$ The equatorial areas of Africa and Papua New Guinea have the highest incidence of all pediatric malignancies (50\%-70\%). ${ }^{11} \mathrm{BL}$ is characterized by chromosomal translocations between c-myc and one of the immunoglobulin loci. ${ }^{12}$ The Epstein-Barr virus has been reported to induce the aggressiveness of $B L$ cells. In the oral cavity, BL occurs most often at the maxilla or the mandible, although other areas may also be affected. Thus, development of an effective therapeutic approach for oral malignant $B L$ is an urgent undertaking. Unfortunately, studies on oral malignant $\mathrm{BL}$ are limited, and the disease remains poorly understood.

It was reported that the roles of Skp2 as a positive regulator of cell cycle in the human tumours, included Burkitt's lymphoma, are to promote the cell growth, induce the cell chemotactic migratory and suppress the cell apoptotic. ${ }^{14}$ Skp-2 protein as a co-activator of kinase inhibitor protein-1 (KIP-1) increases the cell invasion and metastatic through down- regulation of p27Kip-1. ${ }^{16}$ The low accumulation of p27Kip-1 and high expression of Skp-2 protein reveal the cell aggressiveness, poor prognosis, and high mortality. ${ }^{14}$ The effect of Skp-2 AS on an oral malignant Burkitt's lymphoma (Raji) cell through suppression of cell migratory chemotactic and metastatic with down-regulation of metastasis associated protein-1 (MTA-1) and E-cadherin mechanism was investigated. Genetic engineering of Antisense was carried-out to reverse the main function of Skp-2 protein. The aim of study was to examine the effect of antisense p45Skp-2 (Skp-2 AS) suppressed migratory chemotactic and metastasis of oral malignant Burkitt's lymphoma (Raji) cell through down-regulation of MTA-1 and E-cadherin.

\section{METHODS}

The true experimental laboratory with post-test only control group design was confirmed as a type and design of research. This study was approved by the research ethics committee of the Faculty of Dentistry Gadjah Mada University with no. 001385 / KKEP / FKG-UGM / EC / 2018.

\section{Cells and cell cultures}

Raji cells (ATCC CCL-86 B-lymphocyte, USA) were obtained from the Department of Parasitology, Faculty of Medicine Gadjah Mada University. The cells were cultured in Dulbecco's modified Eagle medium (DMEM, Sigma-Aldrich, USA) supplemented with $10 \%$ fetal calf serum, 100 $\mathrm{g} / \mathrm{mL}$ streptomycin, and $100 \mu \mathrm{g} / \mathrm{mL}$ penicillin (Moregate BioTech, Australia). The cultures were incubated at $37^{\circ} \mathrm{C}$ with $5 \% \mathrm{CO}_{2}$ in a $95 \%$ humidified atmosphere.

\section{Antisense oligonucleated experiments (AS)}

AS experiments were performed as described previously. ${ }^{13}$ Two oligonucleotides containing phosphorothioate backbones were synthesized as follows: AS, 5'-TCCTGTGCAT AGCGTCCGCAGGCCC-3' (the AS direction of human Skp2 CDNA nucleotide 22-46) ${ }^{13}$, S, 5'-CCCGGACGCCTGCGATACGTGTCCT-3' (S for $A S)^{13}$. The oligonucleotides were delivered into Raji cell line directly according to the manufacturer's instructions (Antisense technology, Ionis Pharmaceutical, Inc, USA). 


\section{Migration chemotactic assay by Boyden} chamber kit

The Raji cell line ( $5 \times 10^{5}$ cell/kit) was seeded and placed in the upper compartment (each well was $50 \mathrm{l}$ ) and was allowed to migrate through the pores of the membrane into the lower compartment, in which the chemotactic agents [medium + p45Skp-2 AS or p45Skp-2 S or control (SC)] were present.

After an appropriate incubation time (approximately 24 hours), the membrane between the two compartments was fixed with pure methanol and stained with hematoxylin solution, and the number of cells that have migrated to the lower side of the membrane was determined using a light microscope with $40 x$ magnitude.

\section{Western blotting analysis}

The cell lysates were prepared from the Rajitreated cells in a Falcon tissue culture for 48 hours. Briefly, samples containing equal amounts of protein $(70 \mu \mathrm{g}$; using standard procedure for measuring hole protein) were performed an electrophoresis on a SDS-polyacrylamide gel and transferred to a polyvynilidene fluoride (PVDF) membrane (BioRad, Hercules, CA, USA). The filters were blocked in the Trys buffered saline (TBS) containing $5 \%$ non-fat milk powder at $37^{\circ} \mathrm{C}$ for 1 hour, and then incubated with a 1:500 dilution of primary antibodies against the $\mathrm{p} 45 \mathrm{Skp}-2$ protein
(H-435, rabbit polyclonal antibody; Santa Cruz Biotech, USA), MTA-1 protein (AV37737, rabbit polyclonal; Sigma-Aldrich, USA), CDK-2 (E304, rabbit monoclonal antibody, Abcam, USA) and cyclin $\mathrm{E}$ protein (clone HE12, mouse monoclonal antibody, Santa Cruz Biotech, USA).

Detection of HRP-conjugated antibodies was conducted using the enhanced chemiluminescent (ECL) plus kit (Amersham Pharmacia Biotech, UK). Anti-tubulin monoclonal antibody (Zymed laboratories, San Francisco, USA) was used for normalisation of the western blot analysis.

\section{Statistical analysis}

Statistical differences between the means for different groups were evaluated with Stat View 4.5 (Abacus Concepts, Berkeley, CA) software, using the one-way ANOVA test. The significance level was set at $5 \%$ for each analysis.

\section{RESULTS}

\section{Chemotactic migration activity}

The ability of cell migration on each transfected cell was evaluated for $24 \mathrm{~h}$ incubation. Rajitreated cells with p45Skp-2 AS (marked) showed the low ability of cell migration compared with the Skp-2 S and control $(\mathrm{p}<0.05)$ (Figure $1 \mathrm{~A}$ and 1B).

A.

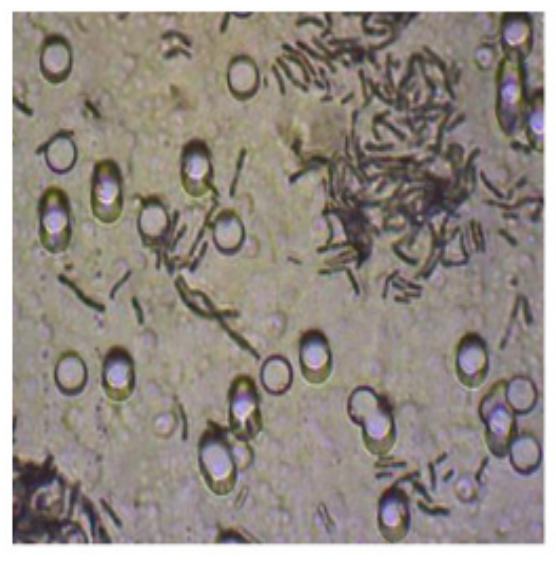

Control (SC)

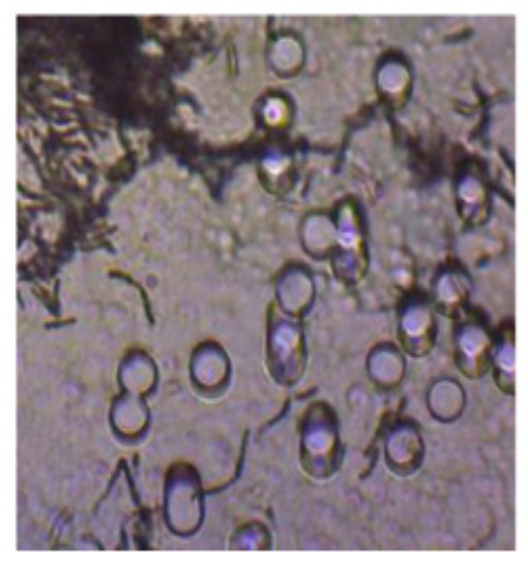

Raji-p45Skp-2 S

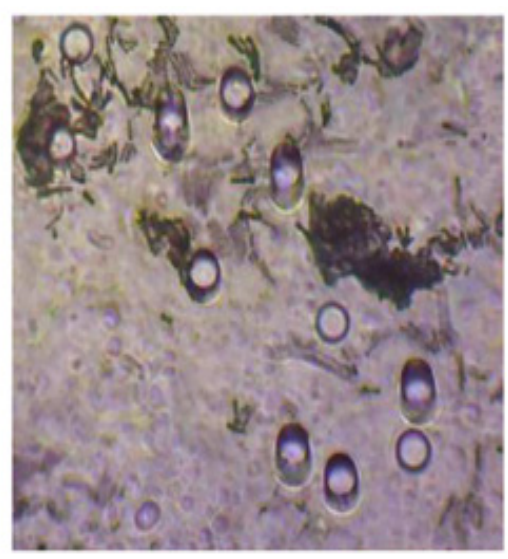

Raji-p45Skp-2 AS

Figure 1. Chemotactic migration activity of Raji cells transfected by oligonucleotides sense (S) and antisense (AS) Skp-2 for 24 hours 
B.

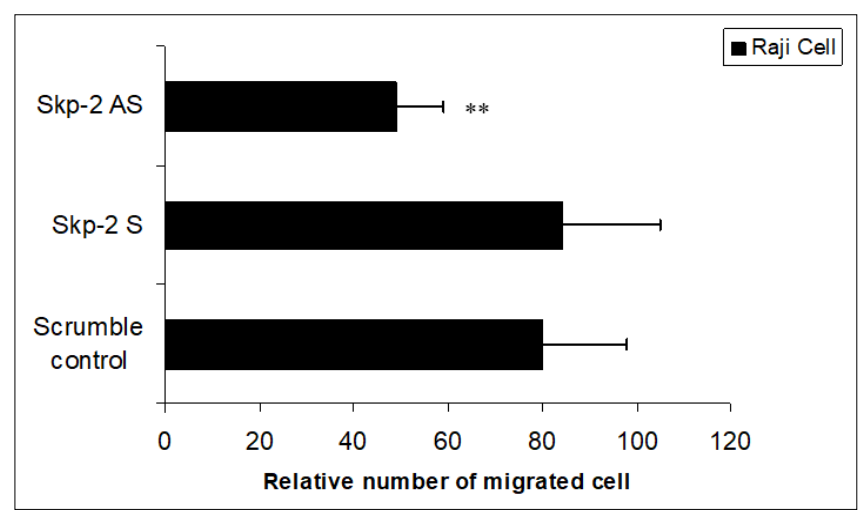

A. Decreased number of Raji cells transfected with Skp-2 AS

B. Relative migrated cell number of Skp-2 AS, S and SC (**; $p=0.001$, one-way ANOVA

\section{Western blotting analysis}

Western blot analysis was used to examine the protein expression of Skp-2, MTA-1, E cadherin and a-tubulin in Raji-transfected with Skp-2 AS, $\mathrm{S}$ or SC. As shown in Figure 2, up-regulation of Skp-2 and E-cadherin protein was detected in
Raji-Skp-2 AS cells compared with that of Skp-2 S. Furthermore, down-regulation of MTA-1 protein was obtained in Skp-2 AS cells. Interestingly, the expression of a-tubulin as an internal control was approximately the same in each Raji-transfected cells.

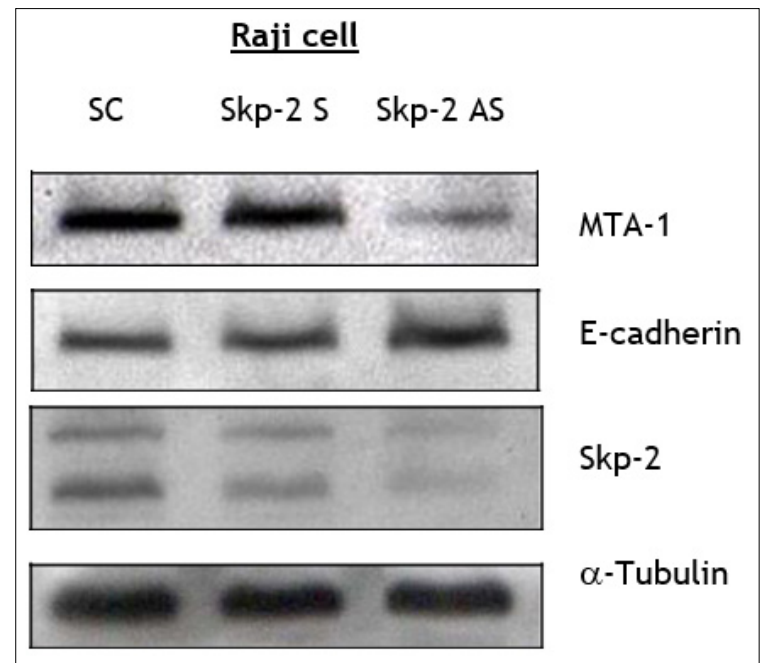

Figure 2. Protein expression of Skp-2, MTA-1, E-cadherin and a-tubulin in Raji cells transfected with Skp-2 AS, S or SC by western blotting analysis

\section{DISCUSSION}

Oligonucleotide-based therapies are advanced novel interventions used in the management of various diseases. Oligonucleotides can be used to modulate gene expression via a range of processes including RNAi, target degradation by RNase $\mathrm{H}$-mediated cleavage, splicing modulation, non-coding RNA inhibition, gene activation and programmed gene editing. The use of oligonucleotides allows for precision and/ or personalized medicine approaches. It can theoretically be designed to selectively target any gene with minimal, or at least predictable, off-target effects. Antisense oligonucleotides (ASODN) have small (18-30) nucleotides, synthetic, single-stranded nucleic acid polymers of diverse chemistries, which can be employed to modulate gene expression via various mechanisms. ${ }^{13}$

In this study, we used phosphorothioate oligonucleotide antisense to Skp-2 because of the function and role of Skp-2 as the tumor promoter gene or protein. Skp2 as a positive regulation of cell cycle can promote the cell migration or invasion. A number of studies have found high levels of Skp2 expression, and its inverse correlation with 
p27 ${ }^{\text {kip1 }}$ level have been observed in lymphomas ${ }^{8}$, oral squamous cell carcinomas ${ }^{3,16}$, lung cancer $^{2}$ and gastric carcinoma. ${ }^{9}$ In the present study, the phosphorotioate oligonucleotide AS and $\mathrm{S}$ strategy was delivered to investigate the activity of Skp-2 on migratory chemotactic and metastasis suppresion in an oral malignant Burkitt's lymphoma cell line (Raji cells) through the down-regulation of MTA-1 and induction of E-cadherin expression. Transfection with Skp-2 AS into Raji cells increased the migratory chemotactic suppression effect (Figure 1 and 2).

These results clearly revealed that cell migratory chemotactic was inhibited by Skp-2 AS effect and not by non-specific effect such as oligonucleotide toxicity. ${ }^{2}$ It has been reported that AS oligonucleotides hybridized to the complementary target mRNA and caused a steric or conformational obstacle for protein translation. As a result, the production of a spesific protein is temporarily inhibited without affecting the expression of other genes and without intervention at the gene level. ${ }^{14}$

Recently, it was reported that the mechanism of action of AS oligonucleotide can be discerned through the Rnase $\mathrm{H}$-dependent oligonucleotide. These appear to induce the degradation of mRNA and the steric-blocker oligonucleotides, which physically prevent or inhibit the progression of splicing or the translational machinery. ${ }^{15}$ Interestingly, oligonucleotide-assisted Rnase $\mathrm{H}$-dependent reduction of targeted RNA expression can be quite efficient, reaching $80-95 \%$ downregulation of protein and mRNA expression. ${ }^{15}$ Recent study reported the relationship between down-regulation of Skp-2 with apoptosis. S-phase kinase protein-2 induction by adenovirus-vector mediated expression of Skp-2 in quiescent cells was followed by apoptosis. ${ }^{2}$ Mice embryonic fibroblasts in Skp-2 deficient mice showed an increased tendency toward spontaneous apoptosis. ${ }^{4}$

However, the actual role of Skp-2 in apoptosis remains unclear. It has been reported that the molecular mechanisms of Skp-2 have an inverse correlation with Kip-1. ${ }^{15}$ Up-regulated of Skp-2 or functional loss of Kip-1 has been implicated in poor prognosis, high carcinogenesis and cancer progression. ${ }^{3}$ In the current research, the mechanism of antisense Skp-2 in suppressing the chemotactic migration, cell growth, metastatic and down regulation of apoptosis via biomolecular mechanism, which can explain why the Skp-2 can promote cel growth and Skp-2 antisense can suppress cell growth.

As expected from the migratory chemotactic inhibitory effect, an increase in protein expression of Skp-2 and E-cadherin was detected in Raji-Skp-2 AS cells strongly suggest that suppression of this migratory cell occured through the target molecule Skp-2 and E-cadherin. It was reported that the loss of $\mathrm{E}$-cadherin expression in association with the epithelial-mesenchymal transition (EMT) occurs frequently during tumor progression, invasion and metastasis. ${ }^{21} \mathrm{E}$-cadherin is particularly active area of research in development and tumorigenesis.

The calcium-dependent interactions among E-cadherin molecules are critical for the formation and maintenance of adherent junctions in areas of epithelial cell-cell contact. Loss of E-cadherin-mediated-adhesion characterises the transition from benign lesions to invasive and metastatic cancer. The mechanism that renders E-cadherin functional is unknown, but it does include phosphorylation of the protein.

Controlled epithelial-mesenchymal conversion is the most important exhibit of E-cadherin's function in development. ${ }^{22}$ Furthermore, the expression of MTA-1 protein was markedly decreased in Raji-Skp-2 AS. This data strongly suggest that Skp-2 AS can suppress Raji cell invasion and metastasis. It was reported The MTA-1 protein contributes to the process of cancer progression and metastasis. ${ }^{17}$ overexpression of MTA-1 protein indicated high proliferation, invasion, metastasis and recurrence in gastric cancer, ${ }^{18-19}$ and also showed rapid formation of tumor angiogenesis and poor survival in lung cancer. ${ }^{20}$

Based on the findings of our 3-years research (2017-2019), we recommend continuing this research by examining other anticancer activities including cell apoptosis, cell cycle arrest, tumirigenesis assay, miRNA assay and in vivo tests. We also have research limitations in the form of research materials that must be obtained abroad and take a long time to be available, research instruments that were limited and queued up, and complicated research permit correspondence. 


\section{CONCLUSION}

p45Skp-2 have an antitumor activity via suppression of migratory chemotactic activity and metastasis on oral Burkitt's lymphoma cells through downregulation of MTA-1 and induction of E-cadherin proteins targeting this molecule could represent a promising new therapeutic approach for this type of cancer.

\section{ACKNOWLEDGMENT}

This research was supported by Hibah Kompetensi grant no. LPPM-UGM/1185/LIT/2017 from Gadjah Mada University, Yogyakarta, Indonesia.

\section{REFERENCES}

1. Pavlides SC, Huang KT, Reid DA, Wu L, Blank SV, Mittal K, et al. Inhibitors of SCF-Skp2/ Cks1 E3 ligase block estrogen-induced growth stimulation and degradation of nuclear p27kip1: Therapeutic potential for endometrial cancer. Endocrinology. 2013; 154(11): 4030-45. DOI: 10.1210/en.2013-1757

2. Su J, Zhou X, Wang L, Yin X, Wang Z. Curcumin inhibits cell growth and invasion and induces apoptosis through down-regulation of Skp2 in pancreatic cancer cells. Am J Cancer Res. 2016; 6(9): 1949-62.

3. Supriatno, Medawati A, Yuletnawati SE. Expression of non-metastatic protein-23 and metastatic associated protein-1 as a molecular target therapy of an oral malignant Burkitt's lymphoma induced by oligonucleotide p27 sense. J Int Dent Med Res. 2020; 13(2): 503-7.

4. Suzuki S, Ohashi N, Kitagawa M. Roles of the Skp2/p27 axis in the progression of chronic nephropathy. Cell Mol Life Sci. 2013; 70(18): 3277-87. DOI: $10.1007 / \mathrm{s} 00018-012-1232-x$

5. Zhou W, Srinivasan S, Nawaz Z, Slingerland JM. ERa, SKP2 and E2F-1 form a feed forward loop driving late ERa targets and G1 cell cycle progression. Oncogene. 2014; 33(18): 234153. DOI: $10.1038 /$ onc. 2013.197

6. Supriatno, Yuletnawati SE. Up-regulation of S-phase kinase associated protein-2 antisense induces cell growth and migration chemotactic suppression and apoptosisin a malignant oral Burkitt's lymphoma cells. Int J Sci Res. 2015;
4(12): $1639-44$

7. Bochis OV, Irimie A, Pichler M, Neagoe IB. The role of Skp2 and its substrate CDKN1B (p27) in colorectal cancer. J Gastrointestin Liver Dis. 2015; 24(2): 225-34. DOI: 10.15403/ jgld.2014.1121.242.skp2

8. Qiu L, Jiabao LV, Chen Y, Wang J, Wu R. Expression of Skp2 and p27kip1 proteins in hypopharyngeal squamous cell carcinoma and its clinical significance. Oncol Lett. 2015; 10(6): 3756-60. DOI: 10.3892/ol.2015.3799

9. Wen $\mathrm{Y}$, Wang $\mathrm{K}$, Yang $\mathrm{K}$. Inhibiting the role of Skp2 suppresses cell proliferation and tumorigenesis of human gastric cancer cells via the upregulation of p27kip1. Mol Med Rep. 2016; 14(4): 3917-24. DOI: 10.3892/ mmr.2016.5676

10. Linch DC. Burkitt lymphoma in adults. $\mathrm{Br}$ J Haematol. 2012; 156(6): 693-703. DOI: 10.1111/j.1365-2141.2011.08877.x

11. Molyneux EM, Rochford R, Griffin B, Newton $R$, Jackson $G$, Menon $G$, et al. Burkitt's lymphoma. Lancet. 2012; 379(9822): 1234-44. DOI: $10.1016 /$ S0140-6736(11)61177-X

12. Salamon D, Adori M, He M, Bonelt P, Severinson E, Kis LL, et al. Type I interferons directly down-regulate $\mathrm{BCL}-6$ in primary and transformed germinal center $B$ cells: Differential regulation in $B$ cell lines derived from endemic or sporadic Burkitt's lymphoma. Cytokine. 2012; 57(3): 360-71. DOI: 10.1016/j. cyto.2011.12.001

13. Roberts TC, Langer R, Wood MJA. Advances in oligonucleotide drug delivery. Nat Rev Drug Discov. 2020; 19(10): 673-94. DOI: 10.1038/ s41573-020-0075-7

14. Supriatno, Yuletnawati SE. Up-regulation of S-phase kinase associated protein- 2 antisense induces cell growth and migration chemotactic suppression and apoptosis in a malignant oral Burkitt's lymphoma cells. Int J Sci Res. 2015, 4(12): 1639-44.

15. Guo S, Booten SL, Watt A, Alvarado L, Freier $S M$, Teckman $J H$, et al. Using antisense technology to develop a novel therapy for a-1 antitrypsin deficient (AATD) liver disease and to model AATD lung disease. Rare Dis. 2014; 2: e28511. DOI: $10.4161 /$ rdis. 28511

16. Evers MM, Toonen LJA, van Roon-Mom WMC. Antisense oligonucleotides in therapy for 
neurodegenerative disorders. Adv Drug Deliv Rev. 2015; 87:90-103

17. Supriatno. S-Phase kinase-associated protein-2 and nuclear factor-kappa beta as molecular targets of oral Burkitt's lymphoma cell induced by quinolinone derivate-vesnarinone. Curr Signal Transduction Ther. 2018. 13: 1-6. DOI: $10.2174 / 1574362413666180912113856$

18. Sen N, Gui B, Kumar R. Role of MTA-1 in cancer progression and metastasis. Cancer Metastasis Rev. 2014; 33(4): 879-89. DOI: 10.1007/ s10555-014-9515-3

19. Yao Y, Feng S, Xiao M, Li Y, Yang L, Gong J. MTA-1 promotes proliferation and invasion in human gastric cancer cells. Onco Targets Ther 2015; 8: 1785-94.

20. Okugawa Y, Mohri Y, Tanaka K, Kawamura $M$, Saigusa $S$, Toiyama $Y$, et al. Metastasisassociated protein is a predictive biomarker for metastasis and recurrence in gastric cancer. Oncol Rep. 2016; 36(4): 1893-900. DOI: 10.3892/or.2016.5054

21. Li SH, Tian H, Yue WM, Li L, Li WJ, Chen ZT. Overexpression of metastasis-associated protein 1 is significantly correlated with tumor angiogenesis and poor survival in patients with early-stage non-small cell lung cancer. Ann Surg Oncol. 2011; 18(7): 2048-56. DOI: 10.1245/s10434-010-1510-5

21. Petrova YI, Schecterson L, Gumbiner BM. Roles for E-cadherin cell surface regulation in cancer. Mol Biol Cell. 2016; 27(21): 3233-44. DOI: $10.1091 / \mathrm{mbc} . \mathrm{E} 16-01-0058$

22. Sun Z, Liu G, Xu N. Does hypermethylation of $C P G$ island in the promoter region of the $\mathrm{E}$-cadherin gene increase the risk of lung cancer? A meta-analysis. Thorac Cancer. 2019; 10(1): 54-59. DOI: $\underline{10.1111 / 1759-7714.12900}$ 